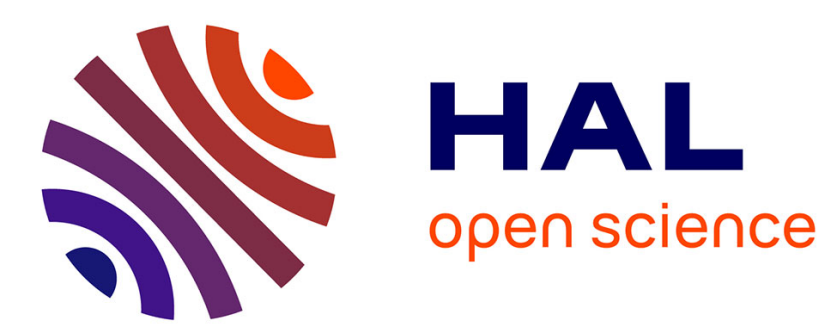

\title{
Une gestion singulière de la crise sanitaire, un système de santé décentralisé sous forte tension
}

Annie Jolivet

\section{To cite this version:}

Annie Jolivet. Une gestion singulière de la crise sanitaire, un système de santé décentralisé sous forte tension. Chronique internationale de l'IRES, 2020, 171, pp.188-208. 10.3917/chii.171.0188 . hal-03405855

\section{HAL Id: hal-03405855 https://hal-cnam.archives-ouvertes.fr/hal-03405855}

Submitted on 27 Oct 2021

HAL is a multi-disciplinary open access archive for the deposit and dissemination of scientific research documents, whether they are published or not. The documents may come from teaching and research institutions in France or abroad, or from public or private research centers.
L'archive ouverte pluridisciplinaire HAL, est destinée au dépôt et à la diffusion de documents scientifiques de niveau recherche, publiés ou non, émanant des établissements d'enseignement et de recherche français ou étrangers, des laboratoires publics ou privés. 


\title{
UNE GESTION SINGULIÈRE DE LA CRISE SANITAIRE, UN SYSTĖME DE SANTÉ DÉCENTRALISÉ SOUS FORTE TENSION
}

\author{
Annie Jolivet
}

\author{
I.R.E.S. | « Chronique Internationale de l'IRES »
}

2020/3 N 171 | pages 188 à 208

ISSN 1285-087X

DOI 10.3917/chii.171.0188

Article disponible en ligne à l'adresse :

https://www.cairn.info/revue-chronique-internationale-de-l-ires-2020-3-page-188.htm

Distribution électronique Cairn.info pour I.R.E.S..

(C) I.R.E.S.. Tous droits réservés pour tous pays.

La reproduction ou représentation de cet article, notamment par photocopie, n'est autorisée que dans les limites des conditions générales d'utilisation du site ou, le cas échéant, des conditions générales de la licence souscrite par votre établissement. Toute autre reproduction ou représentation, en tout ou partie, sous quelque forme et de quelque manière que ce soit, est interdite sauf accord préalable et écrit de l'éditeur, en dehors des cas prévus par la législation en vigueur en France. Il est précisé que son stockage dans une base de données est également interdit. 


\title{
Suède
}

\section{Une gestion singulière de la crise sanitaire, un système de santé décentralisé sous forte tension}

\author{
Annie JOLIVET ${ }^{1}$
}

La Suède a réagi rapidement et au niveau national à l'épidémie provoquée par le SARS-CoV-2. Début février 2020, l'Agence de santé publique suédoise (Folkhälsomyndigheten) classe le Covid19 comme un risque important, activant ainsi le processus de gestion de crise. Alors que fin février, elle considère encore que la probabilité de diffusion dans le pays est faible, sa position évolue au cours des premiers jours de mars avec l'augmentation du nombre de cas. Le 11 mars, date du premier décès, elle formule ses premières recommandations, le 16 mars les premières restrictions. Le 18 mars se tient un Conseil extraordinaire avec un gouvernement réduit.

La stratégie de gestion de la crise sanitaire mise en place à partir du 11 mars se distingue de celle de la plupart des autres pays européens par l'absence de recours à un confinement total et obligatoire. Ce choix est très critiqué et très commenté. Pendant les premières semaines de la crise sanitaire, les taux d'incidence et de mortalité du Covid-19 atteignent en effet des niveaux nettement plus élevés que dans la plupart des autres pays, et surtout trois à quatre fois plus élevés qu'au Danemark et en Norvège et sept fois plus qu'en Finlande.

Cette stratégie repose sur une combinaison de dispositions, certaines relevant de recommandations et d'autres d'obligations assorties de sanctions, et sur la volonté à la fois d'anticiper et de s'inscrire dans une temporalité longue. L'objectif est, comme dans d'autres pays, de limiter le nombre de malades et en particulier le nombre de patients hospitalisés en soins intensifs. Les réformes mises en œuvre en Suède à partir des années 1980 ont privilégié la prise en charge des patients au niveau local par les centres de soins, une spécialisation et une concentration des hôpitaux, et des séjours plus courts avec le développement des soins ambulatoires. La crise sanitaire a donc mis sous pression l'organisation et les capacités du système de santé.

1. Chercheure au Centre d'études de l'emploi et du travail (CEET) - Cnam et au CRTD-Cnam, chercheure associée à l'Ires. 
Après avoir rappelé les principales caractéristiques de l'épidémie de Covid-19, nous mettons en évidence la singularité du mode de gestion et de pilotage des réponses des autorités suédoises. Puis nous soulignons la mise sous tension des capacités sanitaires du pays et la réorganisation des soins pour affronter l'afflux des patients contaminés tout en signalant l'échec des autorités suédoises à protéger les personnes âgées, notamment dans les maisons de retraite. Enfin, nous abordons dans une dernière partie les pénuries de matériels de protection, de tests de dépistage et de produits pharmaceutiques et leurs causes.

\section{L'épidémie de Covid-19 en Suède : une mortalité particulièrement élevée ${ }^{2}$}

L'incidence du Covid-19 est d'abord très marginale avec un petit nombre de cas relevant d'une contamination extérieure à la Suède. Le premier cas est recensé le 31 janvier $2020{ }^{3}$. Au 27 février, sept cas sont identifiés. Jusqu'au début du mois de mars, la progression de la maladie est donc très limitée. La situation évolue rapidement au cours du mois de mars, avec une première accélération modérée, puis une seconde accélération beaucoup plus marquée du nombre de nouveaux cas confirmés (graphique 1). Début avril, 500 cas en moyenne sont confirmés par jour. Un pic est atteint au cours de la seconde quinzaine d'avril avec environ 800 nouveaux cas, puis ce nombre se stabilise jusqu'à la fin du mois de mai. Le nombre de décès progresse de façon exponentielle au cours du mois de mars et atteint un maximum au cours de la première quinzaine d'avril (graphique 2). Au total, entre le 31 janvier et le 10 juin $2020^{4}, 46814$ personnes sont testées positives au SARS-CoV-2, soit 462 cas pour 100000 habitants, 4795 personnes décèdent de Covid-19, soit 47,3 décès pour 100000 habitants.

Début juin, une nouvelle progression très rapide se produit, avec un doublement du nombre de nouveaux cas confirmés en deux semaines (graphique 1), alors qu'une décrue s'observe dans la plupart des autres pays européens. Cette augmentation est liée en partie à l'accroissement du nombre de tests. Toutefois la plupart des cas ne nécessitent pas d'hospitalisation et le nombre de décès poursuit sa décrue.

Les comtés de Stockholm et de Västra Götaland (où se trouve Göteborg, la deuxième ville la plus peuplée du pays) comptent le plus grand nombre de cas. Toutefois la maladie se diffuse dans plusieurs régions au fil des semaines. L'épidémie est donc nationale bien qu'inégalement répartie, avec des rebonds épisodiques dans certaines régions et une variation de semaine en semaine des régions qui connaissent le plus grand nombre de nouveaux cas.

Au 2 octobre 2020, le bilan de l'épidémie s'établit à 94283 personnes (53226 femmes, 41057 hommes) testées positives au Covid-19, soit 931 cas pour 100000 habitants, 5895 décès

2. Selon les informations disponibles au 6 octobre 2020.

3. https://www.krisinformation.se/en/news/2020/january, consulté le 12 juin 2020. La chronologie des événements est retracée à partir des informations fournies par les sites officiels et d'une recension des informations établie au jour le jour sur le site https://www.lasuedeenkit.se/ le-covid-19-en-suede/.

4. Date à laquelle les conférences de presse deviennent bi-hebdomadaires et non plus quotidiennes. 


\section{Graphique 1. Nombre de nouveaux cas quotidiens et moyenne glissante par semaine (février-septembre 2020)}

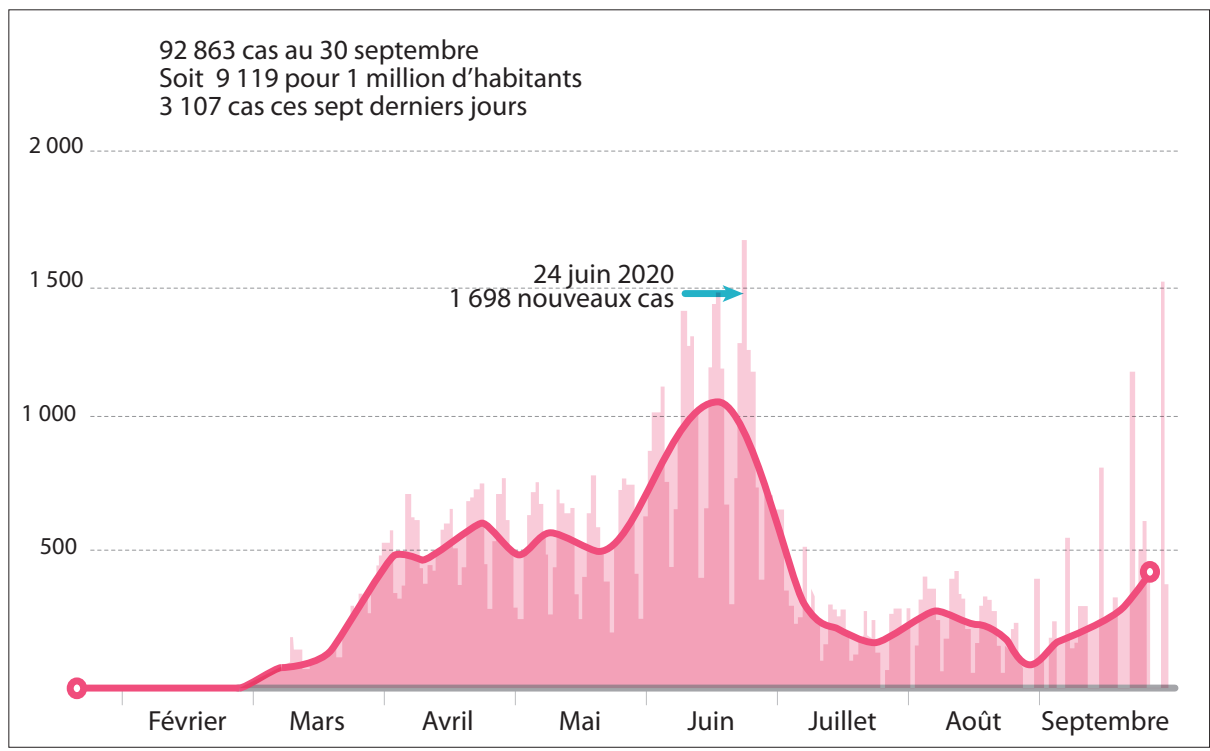

Source : P. Breteau, « Coronavirus : seconde vague, reflux, maîtrise... visualisez l'évolution de l'épidémie dans 140 pays », Le Monde, publié le 27 mars 2020, mis à jour toutes les 24 heures, https://bit.ly/2SIWVWJ, consulté le 5 octobre. À partir des données de l'université Johns-Hopkins. Moyenne des infections par semaine en semaine glissante, du vendredi au vendredi précédent.

\section{Graphique 2. Nombre de décès quotidiens \\ (février-septembre 2020)}

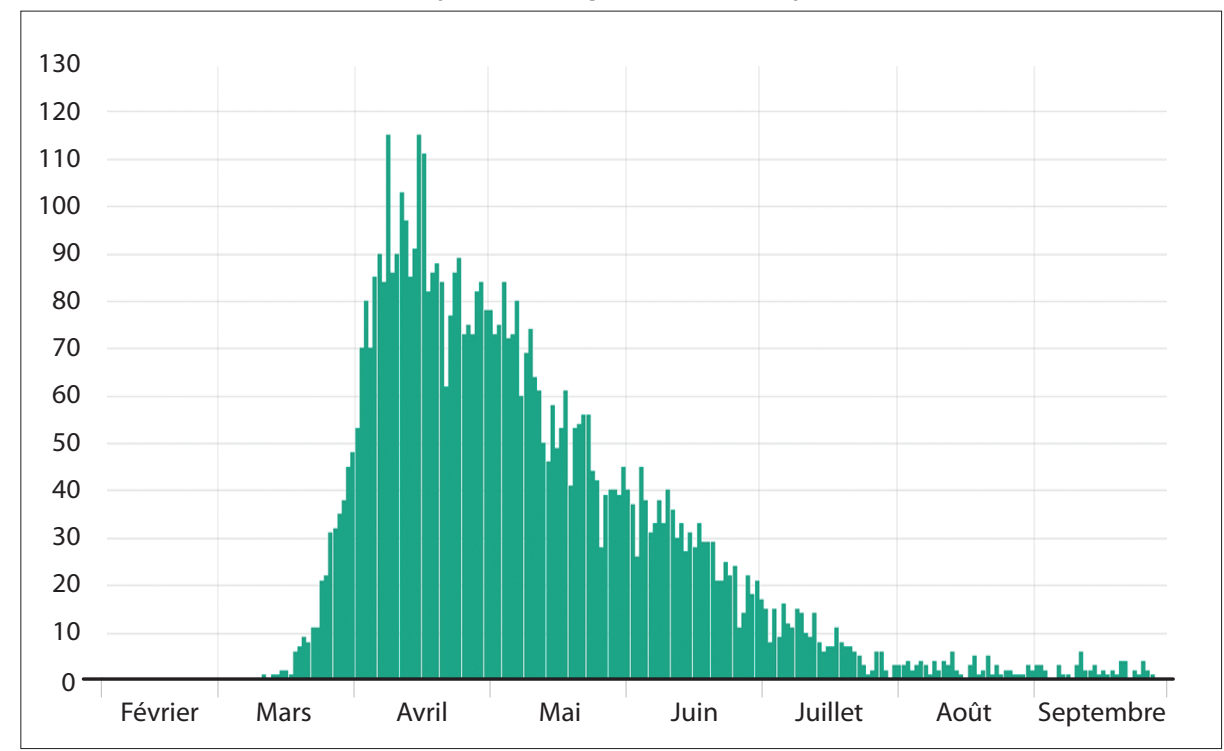

Source : Folkhälsomyndigheten, https://bit.ly/2GCavcb. 
Tableau 1. Comparaison du nombre de cas et de décès pour 5 pays, 6 octobre 2020

\begin{tabular}{|l|c|c|c|}
\hline & $\begin{array}{c}\text { Nombre } \\
\text { de cas }\end{array}$ & $\begin{array}{c}\text { Nombre } \\
\text { de morts }\end{array}$ & $\begin{array}{c}\text { Taux de mortalité } \\
\text { (par million d'habitants) }\end{array}$ \\
\hline Danemark & 30546 & 659 & 113,7 \\
\hline Finlande & 10702 & 346 & 62,7 \\
\hline Norvège & 14605 & 275 & 51,7 \\
\hline Suède & 94283 & 5895 & 578,9 \\
\hline France & 664289 & 32317 & 482,4 \\
\hline
\end{tabular}

Source : Johns Hopkins University, https://coronavirus.jhu.edu/data/mortality.

(2670 femmes, 3225 hommes) soit 58,25 décès pour 100000 habitants, 2611 patients (709 femmes, 1902 hommes) passés par les soins intensifs. Le taux de mortalité reste très supérieur à celui des pays voisins et dépasse même celui de la France (tableau 1).

\section{Une gestion décentralisée et coordonnée de la crise sanitaire, des mesures de restriction limitées}

L'épidémie de Covid-19 déclenche un processus de gestion de crise conforme aux dispositions et aux grands principes de la loi de 2006. Le pilotage de ce processus et les modalités d'action privilégiées expliquent en grande partie la stratégie suédoise.

\section{Les principes de gestion de crise inscrits dans la loi de 2006}

La loi 2006:544 «sur les mesures des communes et des régions avant et pendant les événements extraordinaires en temps de paix et la préparation renforcée » définit une crise, ce qu'elle appelle un " événement extraordinaire », comme tout événement qui s'écarte de la norme, constitue une perturbation sérieuse ou un risque de rupture d'importantes fonctions sociétales, et nécessite une action urgente d'une municipalité ou d'un Landsting (conseil de comté ou de région). Trois principes fondent l'organisation de la gestion de crise : tout acteur responsable d'une activité en temps normal est aussi responsable de cette activité en situation de crise ; l'organisation et le lieu des activités doivent être autant que possible les mêmes en temps normal et en temps de crise ; une crise doit être d'abord traitée là où elle survient, par ceux qui en sont les plus proches.

Comme le soulignent Becker et Bynander (2017), ces trois principes ont pour conséquences une organisation matricielle du système de gestion de crise, croisant domaine de responsabilité et zone territoriale, et la coordination des acteurs impliqués, forcément très nombreux. La loi de 2006 prévoit ainsi six domaines de coordination. Le domaine des matières dangereuses, le plus manifestement concerné par le nouveau coronavirus, regroupe dix agences gouvernementales, dont les trois agences qui sont mobilisées dans la crise sanitaire : le Conseil national de la santé (Socialstyrelsen), l'Agence de santé publique (Folkhälsomyndigheten) et l'Agence de protection civile (Myndigheten för samhällskydd och beredskap, MSB). MSB est l'agence 
gouvernementale chargée en principe de la gestion des crises, de la protection civile, de la défense civile et de la sécurité publique, sauf si la gestion d'une crise ou d'une situation d'urgence est dévolue à une autre agence. Or depuis janvier 2015, Folkhälsomyndigheten a la responsabilité de la lutte contre les maladies transmissibles dans le pays et les menaces internationales à la santé publique. Cette agence gouvernementale qui dépend du ministère de la Santé et des Affaires sociales dispose d'un conseil d'administration indépendant. Sa mission est de produire et de diffuser les connaissances en santé publique à tous les acteurs du système de santé. Elle tient les conférences de presse sur la progression de l'épidémie avec Socialstyrelsen et MSB.

Trois points sont à souligner. Il n'existe pas de loi sur l'état d'urgence qui octroierait des pouvoirs étendus au gouvernement, les procédures normales de discussion au Parlement continuent à s'appliquer. Un ministre ne peut pas décider directement de ce qu'une agence gouvernementale doit faire ou pas, même si elle relève de son champ d'action. Enfin, les autorités territoriales sont dotées de compétences importantes pour faire face aux crises. Dans le cas de la crise sanitaire actuelle, l'essentiel des actions pour ajuster les capacités sanitaires relève des autorités des comtés. Or l'étendue de l'épidémie et les tensions sur le système de santé accroissent la nécessité d'une coordination des acteurs et des ressources ${ }^{5}$. Cela se traduit par la création au niveau national d'une coordination des soins intensifs notamment et par une re-coordination de la gestion des matériels de protection et des tests (voir infra).

\section{Recommandations et interdictions : un partage des tâches entre agence et gouvernement}

La gestion de la crise sanitaire en Suède repose beaucoup plus largement que dans les autres pays sur des recommandations, assez largement suivies, formulées par les experts de l'Agence de santé publique (Folkhälsomyndigheten). Les interdictions sont ponctuelles et du ressort du gouvernement, seul légitime à poser des limites aux libertés individuelles, sous le contrôle du Parlement.

C'est donc Folkhälsomyndigheten qui pose les premières recommandations les 10 et 11 mars. Les personnes qui présentent des premiers symptômes doivent contacter le 1177 (numéro d'appel habituel des services de santé et de soins) et rester chez elles sauf si leur correspondant juge nécessaire qu'elles se rendent à l'hôpital. La consigne est de ne pas aller au centre médical pour éviter la contamination en salle d'attente, de rester chez soi aussi longtemps qu'on se sent malade et de ne retourner à l'école ou au travail qu'après au moins deux jours de guérison. Les visites non indispensables à l'hôpital ou dans les maisons de retraite sont à éviter. Les personnes qui travaillent au contact de personnes âgées ne peuvent aller travailler si elles toussent ou sont enrhumées.

À partir du 11 mars, le gouvernement supprime le jour de carence pour le paiement des congés maladie afin d'inciter les personnes qui commencent à ressentir des symptômes, même bénins, à ne pas se rendre sur leur lieu de travail. Cette mesure prise d'abord pour six semaines est ensuite reconduite. Un certificat médical n'est plus obligatoire pour

5. Ce besoin de coordination a déjà été relevé dans le bilan dressé en 2019 des actions répondant à la canicule exceptionnelle de 2018. 
justifier d'un congé maladie de plus de cinq jours afin de ne pas surcharger les services médicaux. À partir du 12 mars, le gouvernement interdit tous les rassemblements publics de plus de 500 personnes, qu'il s'agisse de manifestations, de concerts, d'événements sportifs, y compris pour les lieux de culte. L'interdiction ne s'applique pas aux fêtes privées, aux bibliothèques, aux salles de sport et aux piscines. Les transports en commun, les centres commerciaux et les marchés restent ouverts. Les prisons suédoises interdisent les visites et les permissions. Le ministère des Affaires étrangères déconseille fortement tous les voyages à l'étranger à partir du 14 mars ${ }^{6}$ et le 19 mars Folkhälsomyndigheten recommande d'éviter les voyages à l'intérieur du territoire suédois pour limiter la propagation du virus.

Les premières recommandations de Folkhälsomyndigheten déclenchent des décisions d'adaptation rapides, certaines anticipant des recommandations ultérieures. De nombreuses entreprises autorisent le travail à domicile, certaines mettent leurs salariés en quarantaine pour deux semaines. Des bibliothèques et salles de sport ferment temporairement, alors qu'elles ne sont pas concernées par une interdiction d'ouverture. Le 16 mars, Folkhälsomyndigheten recommande aux personnes de la région de Stockholm qui peuvent travailler à domicile de le faire. Le même jour, les universités de Stockholm mais aussi de Malmö et de Linköping proposent à leurs étudiants de suivre un enseignement à distance. C'est le lendemain que le gouvernement annonce que les universités, les lycées et les écoles communales pour adultes (komvux) dispenseront leurs cours uniquement à distance à partir du 18 mars. Le Parlement vote le 19 mars une loi permettant au gouvernement de fermer les écoles si cela s'avère nécessaire, sans la recommandation d'un médecin ou d'une autorité.

Le 27 mars, le Premier ministre Stefan Löfven annonce que le seuil d'interdiction des rassemblements publics est abaissé à plus de 50 personnes. Toutefois l'interdiction ne s'étend pas à de nouvelles activités. Löfven souligne dans son discours qu'il est impossible de légiférer sur tout et d'interdire tous les comportements préjudiciables et rappelle que chacun doit prendre ses responsabilités. Le 31 mars est toutefois annoncée l'interdiction, dès le lendemain, de rendre visite aux personnes âgées dans les maisons de retraite (ce qui était auparavant uniquement déconseillé).

La stratégie retenue par Folkhälsomyndigheten repose très largement sur le respect des distances, que ce soit sur le lieu de travail, dans les commerces, dans les transports, dans les réunions et les activités sportives. Comme l'explique Anders Tegnell, épidémiologiste au Folkhälsomyndigheten, dans une interview le 20 avril, la distanciation sociale peut générer les mêmes résultats qu'un confinement, en évitant ses coûts économiques et sociaux. Les écoles maternelles, primaires, les collèges continuent d'accueillir les élèves. Les bars, cafés et restaurants restent ouverts à condition de respecter les distances minimales sous peine de fermeture. Des campagnes de contrôle sont organisées par les communes. La Suède ne prend finalement pas de mesures plus strictes, et ne met pas en place de confinement généralisé et obligatoire.

6. Le Danemark ferme ses frontières dès le 13 mars. Les autres pays voisins de la Suède ferment leurs frontières dans la foulée (Norvège le 16 mars, Lettonie le 17 mars). 


\section{Une stratégie d'immunité collective rapidement très critiquée}

La stratégie privilégiée face à la crise semble être la recherche d'une immunité collective. En témoignent les propos très controversés d'Anders Tegnell, qui pilote la gestion de la crise sanitaire. Il déclare le 8 avril : «Tout épidémiologiste sérieux sait que seule l'immunité collective pourra éventuellement ralentir la propagation du virus. Rien d'autre ne le ralentira à long terme... et nous sommes loin d'un vaccin. » Le recours privilégié à des recommandations est cohérent avec le principe de responsabilité et avec l'idée que ces recommandations seront suivies d'effet. Il y a en effet eu une adaptation des comportements. Ainsi la fréquentation des transports publics a très rapidement et fortement chuté malgré l'absence d'interdiction de se déplacer.

Cependant, plusieurs constats suscitent une critique en Suède de cette stratégie de non-confinement obligatoire, y compris de la part d'autres épidémiologistes : la progression rapide $\mathrm{du}$ nombre de cas déclarés entre la fin du mois de mars et le début du mois d'avril ; le nombre de décès rapidement très supérieur à celui des pays voisins; le nombre important de décès dans les établissements d'accueil de personnes âgées ${ }^{7}$; le ratio beaucoup plus élevé entre le nombre de cas et le nombre d'habitants dans les quartiers nord de la région de Stockholm.
L'association médicale somali-suédoise alerte dès le 24 mars sur le fait que 6 des 15 décès alors recensés dans la région de Stockholm concernent des personnes d'origine somalienne ${ }^{8}$. Des données publiées par l'autorité de santé de la région de Stockholm montrent que le nombre de cas atteint 47 pour 10000 habitants à Rinkeby-Kista et 37 à Spånga-Tensta contre 13 en moyenne 9 . Sont notamment mises en avant les conditions de logement de familles souvent de plus grande taille et les difficultés à comprendre les recommandations en suédois pour des personnes d'origine étrangère, plus fréquentes dans ces quartiers. L'Agence de santé publique diffuse alors des informations en plusieurs langues et certaines autorités locales offrent des possibilités de logement temporaire pour réduire la promiscuité, en particulier en cas de contamination ${ }^{10}$.

Les mesures prises apparaissent incomplètes et insuffisamment strictes : le port du masque n'est pas mis en avant ; peu de tests sont réalisés au début de l'épidémie ; il n'y a pas eu de quarantaine systématique pour des personnes ayant fait des séjours dans les pays touchés par l'épidémie ou présentant des symptômes ; il n’y a pas de traçage des cas constatés. Le 14 avril, dans une tribune du grand quotidien Dagens Nyheter ${ }^{11}$, 22 médecins, virologues et chercheurs dénoncent la stratégie de l'Autorité de santé publique et appellent à des mesures

7. Sur les 5370 décès enregistrés au $1^{\text {er }}$ juillet, $89 \%$ touchent des personnes de 70 ans et plus, et $67 \%$ des personnes de 80 ans et plus. Un peu moins de la moitié des décès sont survenus dans les établissements pour personnes âgées (Socialstyrelsen).

8. "Swedish Coronavirus deaths increase, with concerns about Somali-Swedes in particular », Sveriges Radio, March 24, 2020, https://bit.ly/3nureyH.

9. A.G. Franssen, "Why are there so many coronavirus cases in Stockholm's northern suburbs? », The Local, April 9, 2020, https://bit.ly/3iHgqtx.

10. A.G. Franssen, op. cit.

11. Sous le titre "Folkhälsomyndigheten har misslyckats - nu måste politikerna gripa in » ( L'Autorité de santé publique a échoué - maintenant les politiciens doivent intervenir »), Dagens Nyheter, 24 april 2020, https://bit.ly/2FcgZON. 
plus strictes pour faire baisser le nombre de morts. C'est aussi ce que demandent 2300 médecins et scientifiques, dont le président de la Fondation Nobel, CarlHenrik Heldin, dans une lettre adressée au gouvernement le 25 mars ${ }^{12}$.

\section{Faire face au risque de saturation des services sanitaires}

Le système de santé suédois est un système largement public, décentralisé et hiérarchisé en fonction de la gravité des pathologies à prendre en charge. Son organisation actuelle est le produit de profondes réformes au début des années 1980, puis d'ajustements continus pour contenir la croissance des coûts et améliorer son efficacité (Jolivet, 2014 ; Palier, 2006 ; Desjourdy, 2009 ; Anell et al., 2012). L'une des conséquences les plus visibles est que la Suède a l'un des plus faibles nombres de lits hospitaliers pour 1000 habitants de l'OCDE : 2,2 lits pour 1000 habitants en 2017 (OCDE, 2019). De plus, la répartition des hôpitaux et des lits dépend des choix faits par les Landstings. Le nombre de cas pris en charge par les hôpitaux, et en particulier le nombre de cas en soins intensifs, sont donc une préoccupation majeure.

\section{Un système de santé très décentralisé et des capacités d'hospitalisation au plus juste}

La décentralisation du système de santé a été engagée en 1982 et n'a pas été remise en cause depuis. Le système de santé suédois est régulé au niveau national et administré au niveau local.
Au niveau national, le ministère de la Santé et des Affaires sociales définit les grandes lignes de la politique de santé, fixe les budgets des agences gouvernementales et les subventions accordées aux régions, en coordination avec les agences gouvernementales existantes (voir infra). Huit agences gouvernementales sont directement impliquées dans le système de santé mais sont indépendantes du gouvernement. Ce principe d'indépendance est considéré comme un gage d'efficacité, les décisions des agences n'intégrant pas de considérations politiques. Chaque agence intervient dans un champ de compétences mais elles travaillent en coordination, le Conseil national de la santé (Socialstyrelsen) jouant le rôle de pivot central (schéma 1). C'est Socialstyrelsen qui développe les normes et les procédures de soins, qui s'assure que ces normes sont respectées, qui certifie et supervise l'ensemble des personnels de santé, qui gère les statistiques officielles et les bases de données sur la santé. Parmi ces agences figure l'Agence de santé publique (Folkhälsomyndigheten) qui joue un rôle central dans la gestion de la crise sanitaire.

$\mathrm{Au}$ niveau des comtés ou régions, 21 autorités (Landstings) ont la responsabilité du financement et de la fourniture des soins de santé aux habitants ${ }^{13}$. Au niveau des communes, les 290 municipalités sont en charge des soins et des services aux personnes âgées, aux personnes handicapées, aux personnes qui sortent d'une hospitalisation et sont en charge de la médecine scolaire. Le financement public prédominant $(84 \%$ des

12. D. Robertson, «"They are leading us to catastrophe”: Sweden's coronavirus stoicism begins to jar », The Guardian, March 30, 2020, https://bit.ly/36X3QEr.

13. Les Landstings sont élus tous les 4 ans dans des comtés et des régions comptant entre 60000 et 2 millions d'habitants. Depuis janvier 2020, on ne parle plus que de régions. 


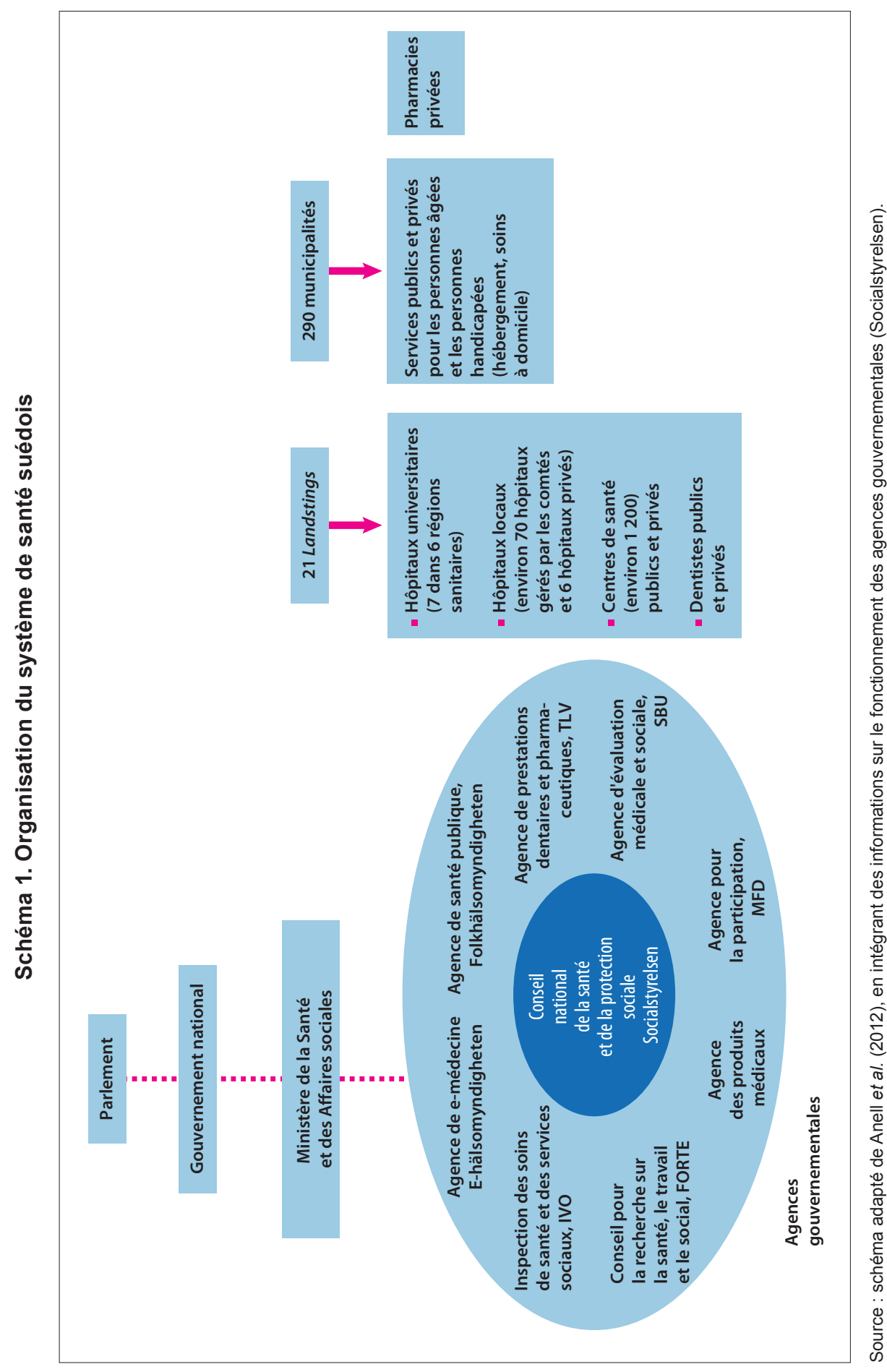


dépenses de santé en $2016{ }^{14}$ ) est presque totalement assuré par les autorités régionales (57\% des dépenses de santé) et les municipalités $(25 \%)$. Les autorités régionales et les municipalités lèvent des taxes locales proportionnelles aux revenus pour financer les services dont elles ont la responsabilité. Le gouvernement central n'intervient donc que très marginalement $(2 \%)$, pour redistribuer des ressources entre les régions et les municipalités selon les besoins ou pour financer des projets spécifiques.

Le partage des tâches entre les autorités régionales, pour tout ce qui relève du traitement médical (hôpitaux et centres de soins primaires) et les municipalités pour tout ce qui relève des soins ambulatoires, de la rééducation, des soins de suite (cliniques, établissements d'accueil, services de soins à domicile) nécessite une coordination des acteurs. Il n'y a pas de relation hiérarchique entre eux, chacun ayant sa propre instance de gouvernance et ses propres champs de compétences. Depuis 2015, les subventions attribuées par le ministère de la Santé et des Affaires sociales sont ciblées sur l'amélioration de la coordination et de la coopération au niveau régional. Par ailleurs, les différents acteurs ont une assez grande marge de manœuvre pour organiser la fourniture des soins, ce qui explique que des variations existent selon les régions.
Toute personne résidant en Suède depuis plus d'un an, immatriculée et inscrite auprès de la Caisse d'assurance sociale (Forsäkringskassan) a accès aux soins et à leur prise en charge par les comtés/régions ${ }^{15}$. Le système de soins est hiérarchisé en fonction de la gravité des pathologies à traiter ${ }^{16}$. Les soins primaires sont délivrés dans les centres de consultation (vårdcentraler) après un appel au 1177 pour obtenir un rendez-vous (dans un délai maximum d'une semaine), être dirigé vers un hôpital ou obtenir une ordonnance ${ }^{17}$. Les hôpitaux locaux (en quasi-totalité propriété des Landstings) assurent des soins hospitaliers courants, principalement programmés et un service d'urgence plus ou moins limité. Enfin, les hôpitaux universitaires concentrent les services très spécialisés et assurent la prise en charge des urgences à tout moment. Les soins intensifs sont fournis à la fois par les hôpitaux universitaires et par deux tiers des 70 hôpitaux régionaux. Les 21 comtés ou régions sont regroupés en six régions en ce qui concerne la santé, pour organiser la coopération entre les fournisseurs de soins (hôpitaux et centres de soins primaires) et pour assurer un haut niveau de soins. Ces six régions sanitaires sont coordonnées par Socialstyrelsen ${ }^{18}$.

Depuis les années 1990, les ajustements ont privilégié la prise en charge des soins en ambulatoire en dehors

14. Chiffres issus de Statistics Sweden, Systems of Health Accounts (SHA) 2001-2016, cités par Glenngård (2020).

15. La participation des patients est plafonnée par période de 12 mois. Des assurances privées sont fournies par des employeurs essentiellement pour garantir un accès plus rapide aux soins ambulatoires et éviter les listes d'attente le cas échéant (Glenngård, 2020). Elles ne financent qu'1 \% des dépenses de santé en 2017.

16. La région de Stockholm a ainsi mis en place une organisation structurée en consistent care chain (Graph, 2017).

17. La Suède est l'un des rares pays où le passage par un centre de consultation joue un rôle de tri, pas de barrière. Les patients sont libres de choisir leur centre de santé, de s'adresser à un spécialiste, et de choisir l'hôpital dans lequel ils souhaitent être traités.

18. https://bit.ly/3jGc8nq. Ces six régions préfiguraient une fusion des comtés qui n'a pas eu lieu. 
Tableau 2. Évolution du nombre de lits et de lits en soins intensifs des hôpitaux

\begin{tabular}{|l|c|c|c|c|c|c|c|c|c|c|}
\hline & 1970 & 1985 & 1990 & 1991 & 1992 & 1995 & 2000 & 2005 & 2010 & 2017 \\
\hline $\begin{array}{l}\text { Lits } \\
\text { pour 1000 } \\
\text { habitants * }\end{array}$ & 15,3 & 14,6 & 12,4 & 11,9 & 7,6 & 4,9 & 3,6 & 2,9 & 2,7 & 2,2 \\
\hline $\begin{array}{l}\text { Lits de soins } \\
\text { intensifs } \\
\text { pour 100000 } \\
\text { habitants ** }\end{array}$ & - & 713,68 & 583,45 & 544,52 & 502,8 & 399 & 310,3 & 266,47 & 249,56 & nd \\
\hline
\end{tabular}

Sources : * Banque mondiale ; OCDE pour 2017.

** OMS (série HFA_478).

des hôpitaux et le développement des centres de soins primaires pour réduire le nombre de personnes s'adressant à l'hôpital et réduire les coûts de santé. Parallèlement, il y a eu pour les hôpitaux un triple mouvement de fusion, de concentration des services spécialisés et de spécialisation des hôpitaux (Anell et al., 2012). Le ratio entre le nombre de lits et la population a ainsi très fortement baissé depuis 1985 (tableau 2). Depuis les années 1990, le nombre de lits a été réduit au-delà de la moitié. Le nombre de lits en soins intensifs a connu la même évolution. Il est passé de 900 lits au début des années 1990 à 576 lits en 2018. Rapporté à la population, le ratio est passé de plus de 963,6 lits pour 100000 habitants

\section{Graphique 3. Nombre de personnes atteintes de Covid-19 en soins intensifs par jour (30 mars-4 octobre 2020)}

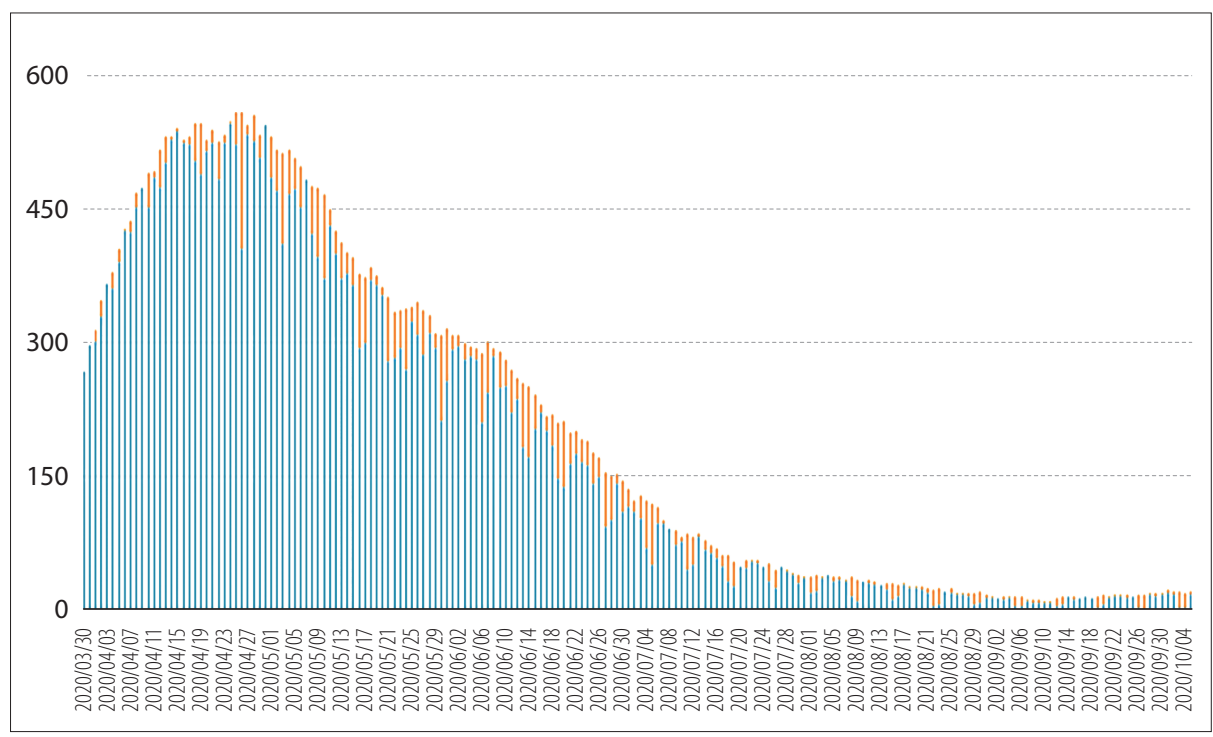

Note : les cas figurant en jaune n'ont pas été comptabilisés le jour de leur admission.

Source : Registre suédois des soins intensifs (Svenska Intensivvårdsregistret, SIR), https://bit.ly/30MVuLD. 
en 1973 à 234,5 en $2014{ }^{19}$ selon les données de l'Organisation mondiale de la santé (OMS), l'un des plus bas en Europe.

La crise sanitaire arrive donc dans un contexte où les capacités hospitalières sont ajustées au plus bas (Graph, 2017 ; IMT, 2020) et où les centres de santé assurent la prise en charge de la plupart des patients. Or entre le 12 avril et le 5 mai, le nombre de personnes atteintes de Covid-19 en soins intensifs dépasse le seuil des 526 lits existant avant l'épidémie (graphique 3). Une augmentation rapide et forte des capacités des établissements est donc nécessaire au fur et à mesure de la propagation de l'épidémie.

\section{L'augmentation anticipée mais pas toujours suffisante des capacités hospitalières}

L'augmentation des capacités est gérée par les Landstings et les hôpitaux eux-mêmes. Ceux-ci augmentent de leur propre initiative le nombre de lits disponibles en déprogrammant les opérations non urgentes et en convertissant des services pour accueillir les malades atteints de Covid-19. L'hôpital universitaire Karolinska, l'un des plus importants du pays, adapte ainsi ses capacités dès la fin du mois de janvier compte tenu des informations dont les médecins disposent via leurs réseaux professionnels. La région de Stockholm annonce le 30 mars que l'hôpital d'Ersta et le Sophiahemmet se réorganisent pour proposer plus de lits. Le 31 mars, l'Akademiska Sjukhuset, hôpital universitaire d'Uppsala, annonce l'ouverture d'un nouveau service de soins intensifs pour les malades du Covid-19 avec neuf lits. Le 7 avril, le représentant du Folkhälsomyndigheten, Anders
Tegnell, indique lors de sa conférence de presse que le nombre de places en soins intensifs atteint 937 lits ( $+80 \%$ en quatre semaines), le 20 avril qu'il est passé à 1098 , avec toujours un taux d'occupation de $80 \%$ (quelle que soit la pathologie), et le 22 avril à 1130 .

Des éléments de coordination supplémentaires sont mis en place au niveau national. Le 20 mars, une mission de coordination des places en soins intensifs est dévolue à Socialstyrelsen par le gouvernement ${ }^{20}$. Par ailleurs, un groupe de travail, composé de médecins spécialistes en maladies infectieuses, d'épidémiologistes et d'analystes de l'Autorité de santé publique livre aux régions le 23 mars des estimations de propagation par zone pour estimer les besoins de soins maximaux en soins hospitaliers et en soins intensifs jour après jour pendant l'épidémie ${ }^{21}$. On retrouve ici le fonctionnement décentralisé et coordonné de la gestion de crise en Suède, avec une coordination nationale renforcée pour une meilleure répartition des ressources.

Trois hôpitaux provisoires sont déployés à Stockholm (Älvsjö), Göteborg et Helsingborg. Le premier est mis en place au centre des expositions de Stockholm, le Stockholmsmässan, à Älvsjö. L'objectif de la région de Stockholm est de doubler la capacité d'accueil de la région en soins intensifs avant qu'elle soit saturée et avant le pic de cas confirmés, avec 600 lits supplémentaires (460 lits pour des patients à symptômes graves et 140 places de soins intensifs avec respirateurs). Les soins et le personnel soignant sont gérés par l'hôpital Karolinska. Cette décision est prise alors que le nombre de cas augmente mais que les hôpitaux de la

19. Selon les données issues de la base HFA (Health for All) de l'OMS, https://bit.ly/34FNEEN.

20. https://bit.ly/3iPEMI3.

21. https://bit.ly/2GCuc3B. 
région n'ont pas encore atteint leur niveau d'alerte ${ }^{22}$. L'hôpital provisoire ouvre ses 10 premiers lits le 6 avril mais n'est toujours pas en activité le 16 avril puisque les sept hôpitaux de la région ne sont pas saturés. Un second hôpital provisoire est construit à Göteborg et ouvert le 8 avril. Il ne s'agit pas de pallier un manque de places dans les hôpitaux de la ville mais de mieux organiser les soins. Le 11 avril, il accueille huit personnes en soins intensifs. Situés dans des zones où l'incidence du Covid-19 est élevée mais où les structures hospitalières sont denses, les hôpitaux provisoires n'accueillent finalement pas autant de patients que prévu. Le 8 juin, la région de Stockholm décide de démonter l'hôpital d'Älvsjö, qui n'a jamais fonctionné.

C'est donc l'augmentation et la réorganisation des capacités des hôpitaux existants qui permettent de faire face à la crise sanitaire. La difficulté reste de prévoir le nombre de patients qui arrivent, en particulier aux urgences. La diffusion du coronavirus n'étant pas uniforme sur tout le territoire, et la répartition géographique des hôpitaux étant inégale, les places créées ne sont pas toujours suffisantes (Löfgren, 2020). Ainsi les hôpitaux de la région de Stockholm ont augmenté très fortement leurs capacités d'accueil et n'ont jamais été débordés par l'afflux de patients. En revanche, la situation est beaucoup plus tendue dans des hôpitaux locaux qui n'ont en temps normal qu'un petit nombre de lits de soins intensifs. La concentration de ces lits et des services spécialisés dans les grands hôpitaux accroît les inégalités d'accès à ces soins selon les régions compte tenu de l'étendue du territoire suédois.

\section{Une réorganisation temporaire des soins et du temps de travail}

La création de lits supplémentaires augmente les besoins en personnel. Or la pénurie et la rotation rapide des personnels soignants sont des problèmes structurels. Le 25 mars, la région de Stockholm, en charge des soins médicaux, demande au personnel à temps partiel d'augmenter son temps de travail et aux retraités du secteur de revenir. Le 30 mars, elle annonce lors d'une conférence de presse que 6500 personnes se sont portées volontaires pour renforcer le personnel soignant actuel. Des personnes mises au chômage économique ont parfois renforcé les effectifs hospitaliers. Alors que la compagnie aérienne Scandinavian Airlines System (SAS) a mis en chômage temporaire 10000 personnes, soit $90 \%$ de ses salariés, l'hôpital universitaire Sophiahemmet a commencé fin mars à former en trois jours un premier groupe de 30 personnes pour apporter une aide aux médecins et aux infirmiers et infirmières ${ }^{23}$. La formation est financée par la fondation Marianne and Marcus Wallenberg.

Le 3 avril, l'organisation patronale des autorités locales et régionales (Sveriges Kommuner och Regioner - SKR, anciennement SALAR) active l'accord de crise pour les soins intensifs dans la région de Stockholm. Cet accord a été négocié après l'expérience de feux de forêts exceptionnels de l'été 2018 qui avaient sursollicité les services d'urgence et soulevé des questions de conditions d'emploi et de compensation salariale. Il a été progressivement signé en 2019 par différentes organisations patronales et syndicales, et par le syndicat des médecins

22. La construction est annoncée le 22 mars pour une ouverture le $1^{\mathrm{er}}$ avril.

23. "Laid-off SAS airline staff offered fast-track healthcare training ", Reuters, March 19, 2020, https://reut.rs/2SESxs4. 
suédois (Sveriges Läkarförbund, SL) le 18 mars $2020{ }^{24}$. L'accord ne peut être déclenché qu'en cas d'urgence, avec l'accord du Conseil régional et de SKR, qui décident à qui il s'applique. Il permet une augmentation du temps de travail jusqu'à 48 heures par semaine, la possibilité de déplacer le personnel soignant vers les communes et les régions qui en ont le plus besoin. En contrepartie, un supplément substantiel de rémunération est versé : $120 \%$ de la rémunération habituelle. Comme l'explique Erik Kjellin, porteparole du syndicat SL, « activer l'accord de crise est extrêmement coûteux », « il n'est activé que si c'est jugé absolument nécessaire » ${ }^{25}$. Au 15 avril, il n'est activé que pour 100 médecins en soins intensifs et uniquement à Stockholm.

Par ailleurs, la concentration des moyens sur les malades du Covid-19 met au jour des besoins nouveaux de prise en charge. Ainsi la ville de Stockholm met en place début avril deux nouveaux centres de soins de courte durée pour les patients qui n'ont plus besoin d'être hospitalisés mais ne peuvent pas être soignés à domicile (Frista Servicehus, Bromsten et Edö Servicehus, Farsta). Deux unités mobiles de dentistes sont mises en place le 13 avril pour traiter les patients atteints de Covid-19 qui ont besoin de soins dentaires d'urgence.

Après avoir passé le pic de contamination, l'enjeu pour les hôpitaux est assez rapidement de reprendre une activité normale, et en particulier de traiter les soins reportés. Le 17 avril, la région de Stockholm commence à s'organiser pour rattraper le retard pris dans les opérations programmées, par exemple les opérations de la cataracte, les mammographies, et autres tests de prévention qui ont dû être repoussés à cause de la crise sanitaire.

\section{Les plus âgés confinés mais mal protégés et peut-être moins soignés}

Les personnes qui décèdent du Covid19 sont essentiellement des personnes âgées de 70 ans et plus, et près de la moitié des personnes décédées résident dans des maisons de retraite. Au 28 avril, $39 \%$ des 7315 personnes de 70 ans et plus testées positives vivent dans des maisons de retraite ${ }^{26}$. Mi-juin, les maisons de retraite de 234 des 290 communes suédoises rapportent être contaminées par le Covid-19.

Le nombre de décès dans les maisons de retraite met en cause la privatisation des établissements accueillant des personnes âgées et la précarité des emplois dans le secteur. Le recours à l'intérim et à des petites durées de travail accroît le risque de contamination et le risque de propagation en augmentant le nombre de personnes qui passent dans un établissement. C'est ce que dénonce le syndicat des salariés des municipalités, Kommunal ${ }^{27}$. Selon l'Association des communes et des régions SKR, un employé municipal sur quatre aux soins des personnes âgées ou handicapées est un travailleur temporaire, et un sur cinq est employé à l'heure

24. Les personnels médicaux, y compris les médecins, sont pour la plupart des salariés des comtés et des municipalités.

25. M. Jankowicz, « Dozens of ICU workers in Stockholm are being paid $220 \%$ of their salary during the coronavirus pandemic, thanks to a "crisis situation" deal that goes back to long before the outbreak », Business Insider, April 15, 2020, https://bit.ly/30Ht2uj.

26. https://bit.ly/2G06a29. Ce taux est de $27 \%$ pour les personnes du même groupe d'âge bénéficiant de l'aide à domicile.

27. https://www.marxist.se/kommunal-hotar-skyddsstopp. 
en novembre 2019 28. Dans la région de Stockholm, ces chiffres atteignent respectivement 43 et $39 \%$. Des employés à l'heure disent leur crainte de propager le virus en passant d'une maison de retraite à l'autre, mais ne pas avoir les moyens de refuser de travailler, avec ou sans symptômes. D'après une enquête menée par le service infectieux de la ville de Stockholm ${ }^{29}$, au moins $30 \%$ du personnel est employé à l'heure ou en intérim dans plus de la moitié des maisons de retraite affectées par le coronavirus. Près d'un employé sur 5 aurait travaillé alors qu'il ressentait des symptômes.

Une autre interrogation porte sur une sélection à l'entrée en soins intensifs qui écarterait davantage les plus âgés dans un contexte de tension lié à l'afflux de patients. Le 24 avril, l'Inspektionen för vård och omsorg (IVO, Inspection de la santé et des soins), alerté par le personnel soignant, lance un audit de l'hôpital universitaire Karolinska. L'hôpital est soupçonné d'avoir refusé les soins intensifs à des patients avec des problèmes rénaux, en dépit d'un nombre suffisant de lits. Parmi les patients de son unité de soins intensifs atteints par le nouveau coronavirus, 9,3\% sont âgés de 70 ans ou plus contre $22,9 \%$ en moyenne dans les unités de soins intensifs. Le 4 mai, l'enquête initiée par IVO s'étend à tous les hôpitaux de Stockholm (Danderyd, Norrtälje, St Göran, Södersjukhuset et Södertälje). Une autre piste est le nonrecours à l'hospitalisation par les établissements d'accueil de personnes âgées.
Finalement, les seules personnes totalement confinées ont été mal protégées de la contamination en raison des conditions d'emploi et de travail dans des établissements pour la plupart privés ${ }^{30}$. Si une plus grande partie de ces personnes avaient été transportée dans les hôpitaux, cela aurait pu accroître la sollicitation des services de soins intensifs. Reste que ces services, en augmentant leur nombre de lits, ont pu afficher un volant de lits disponibles. Le 7 mai, le Premier ministre Stefan Löfven reconnaît que les conditions de travail pour les employés et de vie pour les résidents dans les maisons de retraite doivent être améliorées. Il annonce la mise en place d'une commission d'enquête après la crise, pour passer en revue les mesures prises, faire le bilan, tirer les conclusions des conséquences de la crise, analyser le modèle de gestion suédois, et déterminer ce qu'il faut améliorer. En attendant, l'interdiction de visite aux personnes âgées en établissement est prolongée jusqu'au 31 août, puis jusqu'au $1^{\text {er }}$ octobre.

\section{Pénuries de masques et de certains produits pharmaceutiques}

La Suède a été confrontée au manque de matériel de protection au début de l'épidémie et au manque de tests, même si le gouvernement et les autorités locales n'en ont pas fait état. Alors qu'elle disposait de réserves stratégiques pendant la guerre froide, ces stocks ont été progressivement supprimés/réduits au cours des

28. K. Martinsson, « Var fjärde är tillfälligt anställd i den kommunala äldrevården », Arbetet, 15 April 2020, https://bit.ly/34Dg02o.

29. Information signalée le 23 juin sur https://www.lasuedeenkit.se.

30. La privatisation des établissements accueillant des personnes âgées est une des conséquences des réformes engagées au début des années 1990. 
vingt dernières années ${ }^{31}$. Les stocks de masques dont disposaient les hôpitaux se sont révélés insuffisants face aux besoins massifs. La progression rapide du nombre d'hospitalisations et du nombre d'entrées en soins intensifs a créé des tensions et même des pénuries de certains médicaments utilisés dans le traitement des symptômes.

\section{Des matériels de protection en quantité insuffisante, y compris pour les personnels exposés}

Avec l'afflux de patients et l'augmentation du nombre de lits et de soignants, les stocks de matériels de protection, notamment de masques, s'avèrent insuffisants. Le 11 mars, le manque de masques de protection et de gel désinfectant dans les hôpitaux contraint les dentistes à repousser toutes les opérations non urgentes jusqu'à fin juin. Le 30 mars, les syndicats du personnel soignant font part de l'inquiétude des médecins, infirmiers et infirmières de ne pas être suffisamment protégés en raison du manque de matériel. Les tabliers de protection à manches longues par exemple commencent à manquer et sont remplacés par des tabliers à manches courtes, ce qui oblige à utiliser plus de savon et de gel pour se nettoyer. Les visières doivent aussi être réutilisées et donc nettoyées à l'alcool. Le 17 avril, le syndicat Kommunal demande des équipements de protection adaptés dans les établissements gérés par les municipalités lorsque les personnes effectuent des tâches où elles risquent d'être contaminées (masque, visière, gants et veste de protection à manches longues). L'absence d'équipement de protection ou un niveau de protection insuffisant donne lieu à des arrêts de travail dans de nombreux établissements et à des manifestations d'inquiétude, voire de colère sur les réseaux sociaux ${ }^{32}$ et dans la presse.

Le 4 mai, le syndicat Kommunal porte plainte contre Arbetsmiljöverket ${ }^{33}$ auprès du justitieombudsman $(\mathrm{JO})$, le méditeur du Parlement, pour avoir pris parti pour SKR, l'organisation des communes et des régions suédoises, quant à l'utilisation des masques dans les maisons de retraite. Alors qu'Arbetsmiljöverket avait décidé qu'il était important pour le personnel d'être équipé de masques et de visières, SKR a convoqué Arbetsmiljöverket et trois autres administrations à une réunion, à l'issue de laquelle l'utilisation du masque a été supprimée. Le 29 avril, la mort d'une infirmière de l'hôpital Karolinska (âgée de 39 ans) qui soignait des patients atteints de Covid-19 fait l'objet d'une enquête de la part de la police. Une plainte pour homicide est déposée par le représentant syndical en matière de santé et de sécurité au travail (skyddsombud), qui considère que l'équipement de protection fourni par l'hôpital

31. C. Anderson, H. Pryser Libell, "Finland, "Prepper Nation of the Nordics", isn't worried about masks ", The New York Times, April 5, 2020, https://nyti.ms/3defZ8P. Parmi les pays nordiques, la Finlande a été le seul pays à maintenir des stocks stratégiques et à disposer des réserves nécessaires pour faire face aux besoins suscités par l'épidémie. Voir aussi IMT Sweden (2020).

32. Le groupe Facebook \#vägrasänkahygienkraven Covid-19 (refuser d'abaisser les exigences d'hygiène contre le Covid-19) a dépassé 60000 membres une semaine après sa création.

33. L'Office suédois de l'environnement du travail est une autorité missionnée par le gouvernement et le Parlement suédois pour faire en sorte que les entreprises et les organisations appliquent les lois sur la santé et la sécurité au travail. II vérifie entre autres que les entreprises et les organisations appliquent les lois et les règles en inspectant des sites professionnels à travers toute la Suède. 
n'était pas à la hauteur des normes fixées par Folkhälsomyndigheten.

Des entreprises sont sollicitées ou décident elles-mêmes de réorienter leur activité vers la production de masques et de gel hydroalcoolique. Le 26 mars, le géant H\&M annonce vouloir produire des masques de protection dans ses usines en Chine et les importer ensuite vers l'Europe. Le 8 avril, Essity, une entreprise suédoise spécialisée dans la fabrication de produits d'hygiène, adapte la production de son usine près de Göteborg pour bientôt fournir 3 millions de masques par mois après l'été. Cela se fait dans le cadre d'un dialogue étroit avec le gouvernement et Socialtstyrelsen. Le 13 avril, les autorités annoncent que Perstorp va produire du gel hydroalcoolique et Mölnlycke des masques. Le producteur de vodka Absolut donne aussi de l'éthanol pour permettre la production de désinfectant.
Socialstyrelsen concentre finalement l'achat des équipements de protection individuelle et assure la distribution des masques et du gel hydroalcoolique aux établissements de santé et d'accueil des personnes âgées. Fin mai, l'accès plus important au matériel de protection permet de reprendre les opérations planifiées avant la crise sanitaire. Cette reprise est très variable selon les régions.

\section{Une stratégie tardive et limitée de dépistage au plus fort de l'épidémie}

Le nombre de tests disponibles a pesé sur leur utilisation. Celle-ci a progressé de semaine en semaine (graphique 4), avec plusieurs phases qui s'enchaînent et parfois se recouvrent. Les données publiées par Folkhälsomyndigheten couvrent donc un échantillon dont la composition évolue.

Du début de l'épidémie au 12 mars (semaine 11), les tests de détection du virus sont disponibles en petites quantités

\section{Graphique 4. Nombre de cas testés et de cas positifs par semaine}

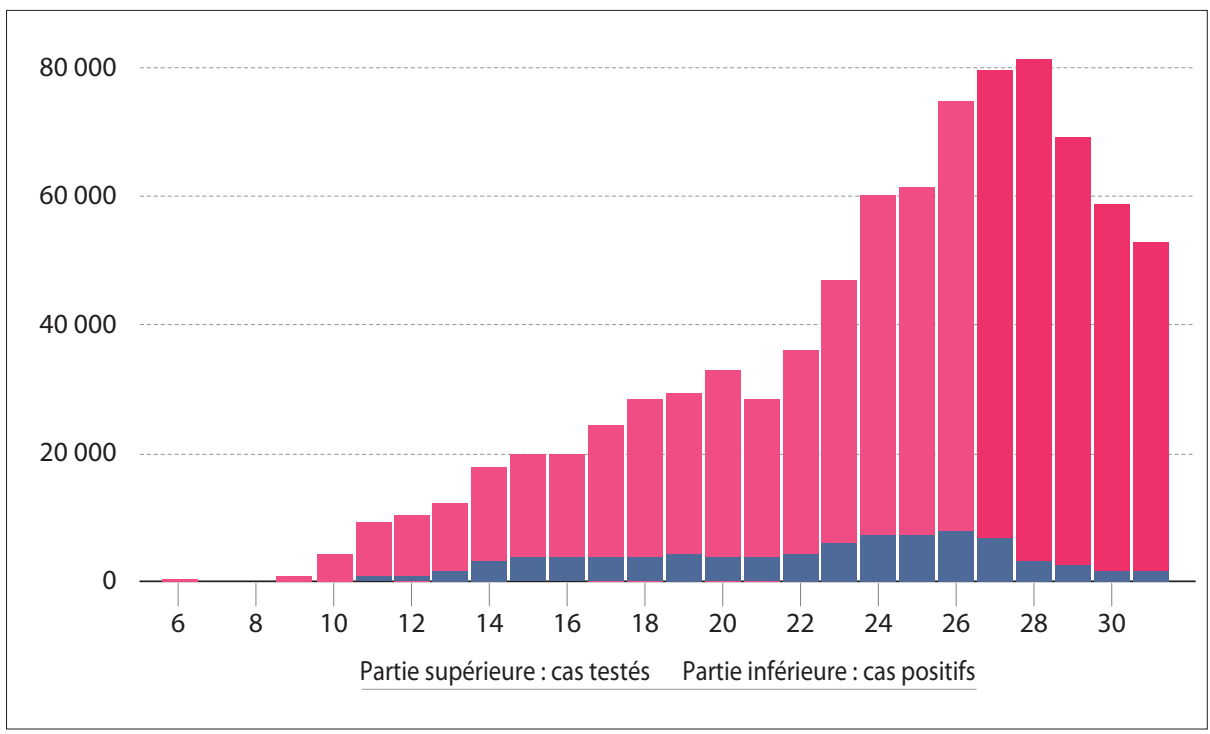

Source : Folkhâlsomyndigheten, rapport hebdomadaire jusqu'au 2 août (semaines 6 à 31). 
et sont utilisés pour les cas importés et leurs contacts. À partir du 13 mars, les tests sont utilisés surtout pour les cas suspects nécessitant une hospitalisation et, dans une certaine mesure, pour les soignants. Le 31 mars (semaine 14), Folkhälsomyndigheten reçoit du gouvernement la mission d'établir une stratégie nationale pour augmenter le nombre de personnes testées. La ministre des Affaires sociales Lena Hallengren parle alors de passer de 10000-12000 tests à 20000-30000 tests par semaine. La stratégie présentée le 17 avril (semaine 16) se fixe l'objectif encore plus ambitieux de procéder à 50000-100000 tests par semaine, et de tester davantage de personnes travaillant dans des activités essentielles afin qu'elles puissent retourner travailler dès que possible. Trois groupes prioritaires sont définis : 1) les personnes à risque et hospitalisées ; 2) le personnel soignant ; 3) les professions critiques pour le bon fonctionnement de la société ${ }^{34}$. Pour ces personnes, le test est pris en charge par l'État et non par les Landstings ${ }^{35}$. À partir du 19 mai, les personnes qui ont un rendez-vous dans un centre médical (vårdcentral) peuvent se faire tester. Le 5 juin (semaine 23), le gouvernement charge Folkhälsomyndigheten et les Landstings d'assurer des flux de tests à grande échelle. La capacité est progressivement augmentée, ce qui accroît à la fois le nombre de cas confirmés et la proportion de cas signalés qui n'ont pas besoin de soins hospitaliers. Le 26 mai, l'objectif n'est toujours pas atteint : 28800 tests de dépistage sont effectués au cours de la semaine précédente (semaine 21), seulement 4000 de plus que la semaine 20 (graphique 4). Les régions disent avoir rencontré des problèmes de logistique. De fait, elles testent plus ou moins largement. La région de Stockholm commence à tester le troisième groupe prioritaire et compte effectuer 40000 tests cette semaine.

Les tests anticorps arrivent plus tardivement, sans que la durée de l'immunité soit bien connue. Fin avril, les tests anticorps disponibles sur Internet sont jugés non fiables et sont interdits par l'Agence du médicament et des produits de santé (Läkemedelsverket). L'objectif affiché est d'atteindre 100000 personnes testées par semaine à la mi-mai, par exemple en mettant en place des drive-in. La chaîne de pharmacie Apotea commence à vendre des tests anticorps le $1^{\text {er }}$ juin.

Le 3 juin, Harriet Wallberg achève la mission de trois semaines confiée par le gouvernement pour coordonner les tests dans le pays. Elle recommande de tester massivement pour diminuer la propagation du SARS-CoV-2, afin d'être mieux préparés à la deuxième vague qui viendrait à l'automne.

\section{Au pic de l'épidémie, des pénuries de médicaments ou matériels de soins intensifs}

Le pic des hospitalisations en soins intensifs est atteint entre le 8 avril et le 5 mai. Il fait craindre notamment une pénurie de Propofol, utilisé pour endormir les patients sous respirateur, au cours du week-end de Pâques. Le 10 avril, l'Agence du médicament et des produits de santé (Läkemedelsverket) approuve qu'un médicament vétérinaire, de la

34. Une liste de 12 secteurs clefs a été identifiée le 20 mars par le gouvernement. MSB a ensuite identifié 39 professions le 26 mars. Il s'agit entres autres de la police, de l'armée, des services d'urgence, de l'approvisionnement énergétique et alimentaire.

35. Le 9 avril, le gouvernement autorise l'Institut vétérinaire à analyser les tests humains, en appui au système de santé. 
même composition, soit utilisé comme substitut. Mi-mai, l'hôpital de Blekinge indique qu'il opère désormais uniquement sous anesthésie locale ou péridurale, pour réserver l'usage du médicament Propofol aux patients Covid-19 qui en ont besoin en soins intensifs. L'objectif est de ne pas repousser trop d'opérations dans le futur, pour éviter une file d'attente trop longue une fois la crise du coronavirus passée.

Ces pénuries remettent en lumière le changement d'organisation intervenu en 2009 pour l'approvisionnement des établissements de santé et de soins en produits pharmaceutiques (Anell et al., 2012). Jusqu'en 2009, cet approvisionnement relevait de la pharmacie d'État qui détenait un monopole. Avec l'ouverture à la concurrence, les huit pharmacies centrales ont été dissoutes. Deux questions liées sont posées : la possibilité ou non de produire rapidement sur le sol suédois pour répondre à l'augmentation rapide des besoins et l'existence d'un acteur qui puisse commander pour l'ensemble des acteurs locaux, de façon à limiter la hausse des prix voire contribuer à ce qu'une production se mette en route.

La pénurie touche également le matériel de réanimation en raison du nombre de malades admis en soins intensifs et $\mathrm{du}$ doublement du nombre de places ouvertes dans ces services. Les salariés de Scania, constructeur automobile, dont l'usine était à l'arrêt sont ainsi envoyés travailler dans une entreprise qui produit des respirateurs.

\section{Conclusion}

L'afflux de patients entre la mi-mars et la mi-avril a mis sous tension très forte le système de santé. Les hôpitaux ont accueilli directement beaucoup des personnes atteintes du Covid-19, en particulier dans les services de soins intensifs. La montée en charge a été rapide, forte et a nécessité des réorganisations importantes sur plusieurs semaines, une augmentation du temps de travail et le recours à du personnel supplémentaire non formé a priori. Cette montée en charge a été d'autant plus coûteuse que le nombre de lits avait été ajusté à un niveau minimal, résultat d'une longue série de réformes et de réorganisations. L'activation de l'accord de crise et la création d'hôpitaux provisoires n'ont permis que très marginalement de faire face à la crise sanitaire.

Le choix de ne pas confiner totalement et de façon obligatoire l'ensemble de la population apparaît cohérent avec le système de gestion de crise suédois, très décentralisé et reposant sur un principe de responsabilité individuelle. Les recommandations ont eu un impact fort et rapide sur les comportements. Il y a néanmoins eu quelques mesures contraignantes (confinement des personnes âgées, distances dans les lieux restés ouverts et les transports publics), avec des contrôles et des sanctions (dans le cas de non-respect des distances dans/par les bars, cafés, restaurants). L'objectif est davantage d'adapter que de contraindre les comportements.

La volonté d'anticiper et de gérer la crise sur un temps long a pris appui sur le partage clair et connu des responsabilités entre les agences gouvernementales (Folfhälsomyndigheten au premier chef) et les acteurs locaux. La loi de 2006 a donné en effet un cadre à la définition d'une stratégie de gestion de la crise sanitaire. L'expérience dont dispose la Suède en matière d'événements de grande ampleur est cependant plutôt récente (les 
exceptionnels feux de forêt de 2018, les épisodes de canicule). Cette crise sanitaire a rendu nécessaire un certain degré de centralisation pour gérer les pénuries, en rupture avec la décentralisation habituellement privilégiée : Socialstyrelsen a ainsi pris en charge la coordination entre régions des places de soins intensifs et l'achat des matériels de protection.

La crise sanitaire a révélé plusieurs points noirs. Il a d'abord été impossible de protéger les personnels soignants et les personnes susceptibles d'être exposées en raison de l'absence de stocks stratégiques. La difficulté à se procurer rapidement et en quantité des produits qui n'ont pas fait l'objet d'un stockage suffisant, dans un contexte d'explosion de la demande mondiale et de concurrence exacerbée entre les pays, interroge les conditions d'approvisionnement mises en place depuis la fin des années 2000. La très forte mortalité dans les établissements pour personnes âgées a mis en cause les conditions d'emploi précaires notamment liées à la privatisation. Enfin, la mise sous tension du système de santé a remis en lumière les besoins en personnel soignant des hôpitaux.

D'abord prévue par le gouvernement après la crise, la commission d'enquête sur la gestion de la crise sanitaire est finalement mise en place le 30 juin, à la demande de plusieurs partis d'opposition. Un premier rapport est prévu le 30 novembre 2020, deux autres s'échelonneront jusqu'à fin février 2022. Cette commission devrait fournir un bilan détaillé des dispositions mises en place et une évaluation de la gestion de la crise sanitaire par le gouvernement, les agences gouvernementales, les autorités des régions et des communes.

Achevé de rédiger le 26 octobre.

\section{Sources :}

Anell A., Glenngård A.H., Merkur S. (2012), "Sweden health system review ", Health Systems in Transition, vol. 14, $n^{\circ}$ 5, p. 1-159, https://pubmed.ncbi.nlm.nih.gov/22894859/.

Becker P., Bynander F. (2017), " The system for crisis management in Sweden: Collaborative, conformist, contradictory ", in Madu C.N., Kuei C. (Eds.), Handbook of Disaster Risk Reduction \& Management, Singapore, World Scientific, p. 69-95, https:// doi.org/10.1142/10392.

Desjourdy A. (2009), « Les réformes de santé en Suède : quelles leçons pour le Québec ? ", Cahiers de recherche en politique appliquée, vol. 2, n 3, p. 17-51, https://bit.ly/2Fc0CBf.

Glenngård A.H. (2020), "Country Profiles: Sweden ", International Health Care System Profiles, The Commonwealth Fund, June 5, https://bit.ly/33GRe1T.

Graph (2017), « Le système de santé suédois ", Gestions hospitalières, $n^{\circ} 568$, septembre, p. 403-405, http://www.le-graph.com/ images/publications_graph/graph_suede.pdf.

IMT Sweden (2020), « The slipping mask of Swedish capitalism ", Revolution, May $1^{\text {st }}$, https://www.marxist.com/the-slipping-maskof-swedish-capitalism.htm.

Jolivet A. (2014), « Suède : des réformes et des ajustements bien antérieurs à la crise ", $\mathrm{n}^{\circ}$ spécial, "Santé, éducation : services publics dans la tourmente ", Chronique internationale de l'IRES, $\mathrm{n}^{\circ} 148$, décembre, p. 140 150, https://goo.gl/VVD5qK.

Löfgren E. (2020), «"The biggest challenge of our time": How Sweden doubled intensive care capacity amid Covid-19 pandemic », The Local, June 23, https://bit.ly/3jHflO1.

OCDE (2019), Panorama de la santé 2019: Les indicateurs de l'OCDE, Paris, Éditions de I'OCDE, https://doi.org/10.1787/5f5b6833-fr.

Palier B. (2006), « Un système régionalisé en constante évolution : le cas suédois ", Les Tribunes de la santé, $\mathrm{n}^{\circ} 3$, p. 29-36, https:// www.cairn.info/revue-les-tribunes-de-lasante1-2006-3-page-29.htm.

Sites

Agence de santé publique - Folkhälsomyndigheten : https://bit.ly/3luh3Z1

Conseil national de la santé - Socialstyrelsen : https://www.socialstyrelsen.se 
SUÈDE

Svenska intensivvårdsregistret : https://bit. Centre des Liaisons européennes et interIy/30MVuLD.

Gouvernement central : www.regeringen.se

https://www.lasuedeenkit.se

nationales de Sécurité sociale - CLEISS (20-

20), Le système de santé suédois, https://www.

cleiss.fr/docs/systemes-de-sante/suede.html. 